\title{
LESIONES GÁSTRICAS, HEPÁTICAS Y RENALES EN EL SÍNDROME DE ÚLCERAS GÁSTRICAS EN EQUINOS PURA SANGRE DE CARRERA
}

\author{
Gastric, Liverand Renal Lesions Associated to the Syndrome of Gastric \\ UlCERS IN ThOROUghbred HorSes
}
Abelardo Morales B. ${ }^{1,2}$, Francisco García G. ${ }^{1}$, Víctor Bermúdez G. ${ }^{1}$, Elías Sogbe M. ${ }^{1}$, Miguel Perdigón M. ${ }^{3}$

\section{ResUMen}

\begin{abstract}
El objetivo del estudio fue describir lesiones gástricas, hepáticas y renales en caballos Pura Sangre de Carreras enmarcadas en el síndrome ulceroso gástrico equino. Se trabajó con 30 ejemplares Pura Sangre de Carrera con historia de síndrome ulceroso gástrico y cólico recurrentes entre 2006 y 2007, en el Hipódromo «La Rinconada», Caracas, Venezuela. A los animales se les practicó la eutanasia y se colectó nuestras de tejido gástrico, hepático y renal. Las muestras fueron procesadas por los métodos histológicos convencionales. En el estómago se observó mucosa intacta, pero con áreas de hiperemia y/o hiperqueratosis en 6 casos, pequeñas erosiones multifocales o úlceras en 30 casos, úlceras grandes multifocales con regiones extensas de erosión en 12 casos, y úlceras extensivas con áreas de exposición de la submucosa en 6 casos. La evaluación macroscópica del hígado evidenció icteria en 22 casos, así como hepatomegalia y patrón lobulillar en 18 casos. Los riñones mostraron congestión severa en todos los casos, pérdida de la relación córtico medular en 20 casos, necrosis cortical 18 casos, necrosis tubular aguda en todos los casos, necrosis papilar confluente en 6 casos y adherencia de la cápsula con fibroplasia intersticial en 4 casos. Las lesiones observadas en la mucosa gástrica, hígado y riñón estuvieron asociadas con terapéuticas prolongadas con antiinflamatorios no esteroideos.
\end{abstract}

Palabras clave: equinos, patología, gastritis, digestivo, hígado, renal, Síndrome de Úlceras Gástricas

\section{Abstract}

The aim of this study was to describe gastric, hepatic, and renal lesions in Thoroughbred Racing horses associated to the equine gastric ulcer syndrome. Thirty horses with a history of gastric ulcer syndrome and recurrent colic during 2006 to 2007 at the Race Track «La Rinconada», Caracas, Venezuela were evaluated. Horses were

\footnotetext{
${ }^{1}$ Departamento de Patología Veterinaria, Facultad de Ciencias Veterinarias, Universidad Central de Venezuela, Maracay, Estado Aragua, Venezuela

${ }^{2}$ E-mail: aamorales13@hotmail.com

${ }^{3}$ Departamento de Patología, Instituto Nacional de Hipódromos «La Rinconada», Caracas, Venezuela
} 
euthanized and the necropsy was conducted. Tissue samples were collected from stomach, liver and kidney, and processed by conventional histological methods. In the stomach, the mucosa was intact but areas of hyperaemia and hyperkeratosis were observed in 6 cases, multifocal small erosions or ulcers in 6 cases, multifocal large ulcers with areas of extensive erosion in 12 cases and extensive areas of ulceration with exposure of the submucosa in 6 cases. The macroscopic evaluation of the liver showed evident hepatomegaly in 22 cases and lobular pattern in 18 cases. The kidneys showed severe congestion in all cases, loss of cortico-medullar ratio in 20 cases, cortical necrosis in 18 cases, acute tubular necrosis in all cases, papillary necrosis in 6 cases, and adherence of the capsule with interstitial fibroplasia in 4 cases. Lesions in the gastric mucosa, liver and kidney were associated with extended treatment with non-steroidal anti-inflammatory drugs.

Key words : equine, pathology, gastritis, digestive, liver, kidney, equine gastric ulcer syndrome

\section{INTRODUCCIÓN}

El Síndrome de Úlceras Gástricas en Equinos (SUGE) es una patología común en este tipo de animales, y se caracteriza por presentar inflamación y ulceración de la mucosa gástrica, gastritis aguda o crónica, erosión gástrica, ulceración péptica y gastropatía (Murray, 1994, Merrit, 2003).

La etiología del SUGE se considera multifactorial y está asociada a factores de estrés (liberación de catecolaminas, cortisol), ejercicio intenso (acidosis metabólica no compensada), manejo, dietas y calidad nutricional (disposición de nutrientes y requerimientos), enfermedades subyacentes (enfermedades infecciosas), y uso de antinflamatorios no esteroideos (AINES) como fenilbutazona, y corticoides como la dexametasona (Andrews et al., 2005). En casos clínicos, se ha aislado en muestras de heces a Helicobacter equorum, una nueva especie del género Helicobacter (Moyaert et al., 2007).

SUGE está bien documentado (Murray et al., 1994). La incidencia de úlceras gástricas en caballos de carrera que se encuentran en actividades de competencia puede exceder el 90\% (Murray, 1994). La presentación clínica varía de acuerdo a la magnitud de la lesión, siendo los principales signos clínicos la anorexia, bruxismo, ptialismo, diarrea, perforación gástrica, peritonitis, síndrome de emaciación, cólicos recurrentes, y disminución del rendimiento atlético, entre otros. Asimismo, existe una presentación clínica asintomática, que pese a la presencia de algunas lesiones no muestra signos clínicos. Las lesiones gástricas se han descrito en la literatura; sin embargo, no existen reportes de lesiones en otros tejidos u órganos asociados a este síndrome. El objetivo de este trabajo fue describir lesiones gástricas, hepáticas y renales en caballos Pura Sangre de Carreras enmarcadas en el síndrome ulceroso gástrico equino.

\section{Materiales y Métodos}

Se realizó un estudio clínico-patológico a 30 ejemplares Pura Sangre de Carrera (PSC) (12 machos y 18 hembras), entre 2 y 6 años de edad, que presentaban historia de síndrome ulceroso gástrico, cólico recurrente e intolerancia al ejercicio. El tratamiento de rutina era $2.2 \mathrm{mg} / \mathrm{kg}$ de fenilbutazona y 1 $\mathrm{mg} / \mathrm{kg}$ de furosemida por tres meses. Estos animales no se llegaron a recuperar, fueron sacrificados (Melling y Alder, 1998) y la necropsia (Aluja y Constantino, 2002) se realizó en el Hospital Veterinario del Instituto Nacional de Hipódromos (INH) «La Rinconada», Caracas-Venezuela, entre los años 2006 y 2007. 


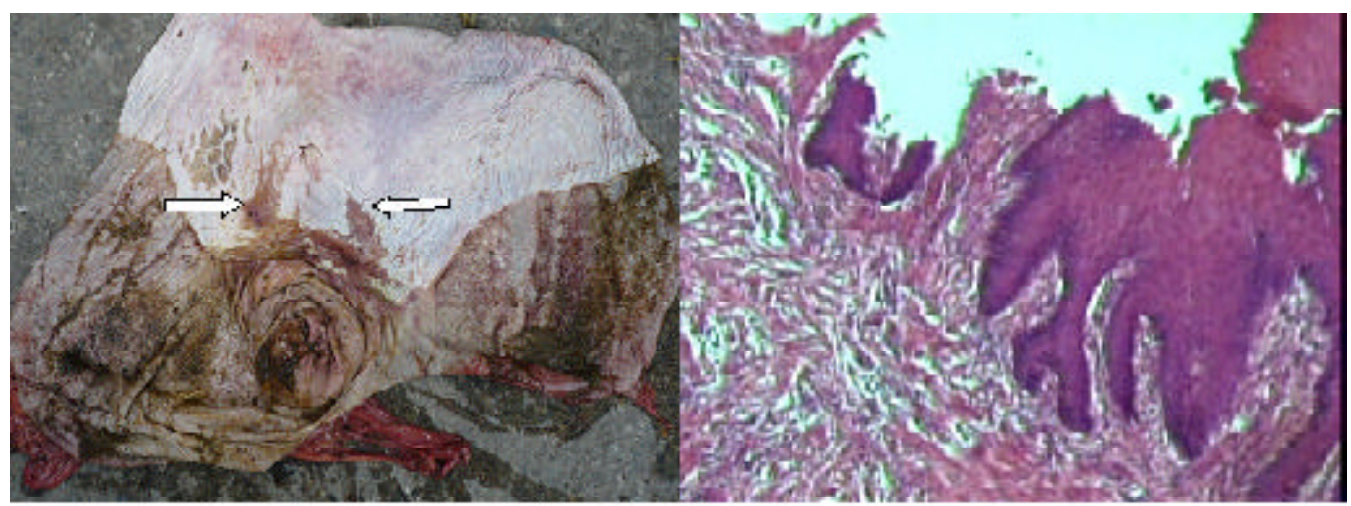

(a)

(b)

Figura 1. Mucosa gástrica con síndrome ulceroso gástrico severo (Grado 4), según la clasificación de Merritt (2003). (a) Vista macroscópica. Las flechas señalan úlceras extensivas con áreas de exposición de la submucosa. (b) En la microfotografía se observa exposición del corion, acompañada de infiltrado mononuclear linfocítico y plasmocítico en la lámina propia, e hiperqueratosis paraqueratótica severa con fibroplasia focal (H\&E, 20X)

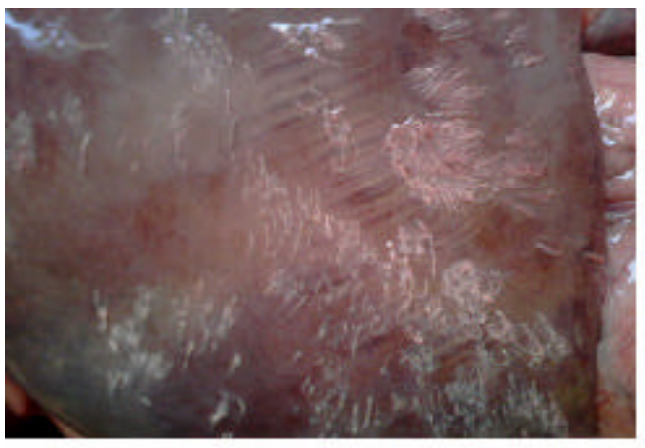

(a)

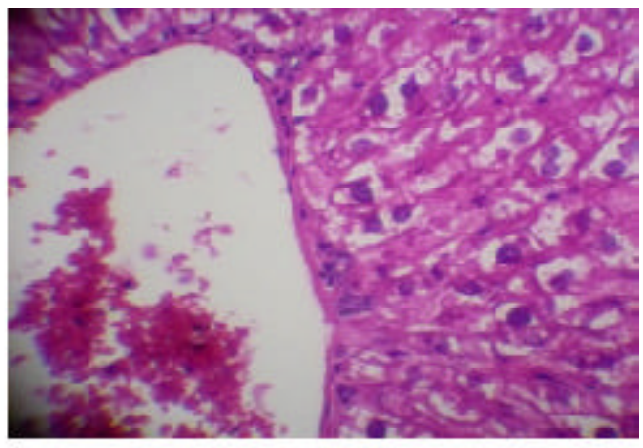

(b)

Figura 2. Hígado de equino con síndrome ulceroso gástrico en grado 4, según la clasificación de Merritt (2003). (a) Ictericia, hepatomegalia, patrón lobulillar, focos de necrosis de coagulación confluente con respuesta micronodular y fibroplasia focalizada. (b) Ictericia, necrosis de hepatocitos en zona periacinar, respuesta micronodular, y degeneración grasa (H\&E, 40X)

Muestras de tejido gástrico, hepático y renal fueron colectadas para el estudio histopatológico. Las muestras se procesaron siguiendo los métodos histológicos convencionales (fijación con formol al 10\%, deshidratación/ aclaramiento, inclusión en parafina, cortes al micrótomo y tinción con Hematoxilina y Eosina) (Banks, 1996).
La evaluación macro y microscópica de la mucosa gástrica se realizó de acuerdo a la clasificación de Merrit (2003), donde Grado 1: Mucosa intacta pero con áreas de hiperemia y/o hiperqueratosis; Grado 2: Pequeñas erosiones multifocales o úlceras; Grado 3: Úlceras grandes multifocales, con regiones extensas de erosión; y Grado 4: Úlceras extensas con áreas de exposición de la submucosa. 


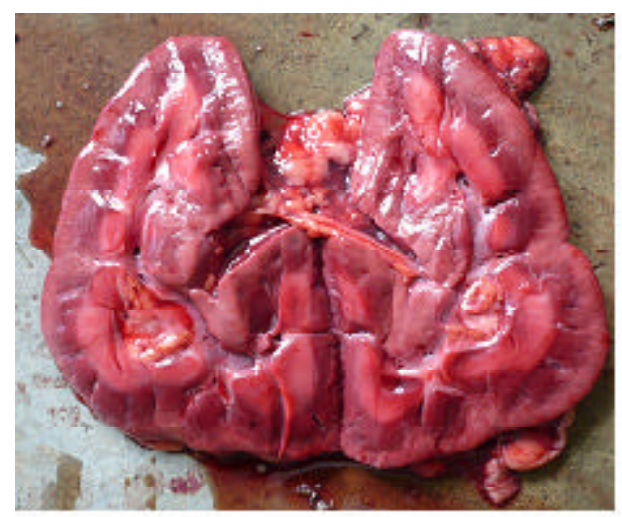

(a)

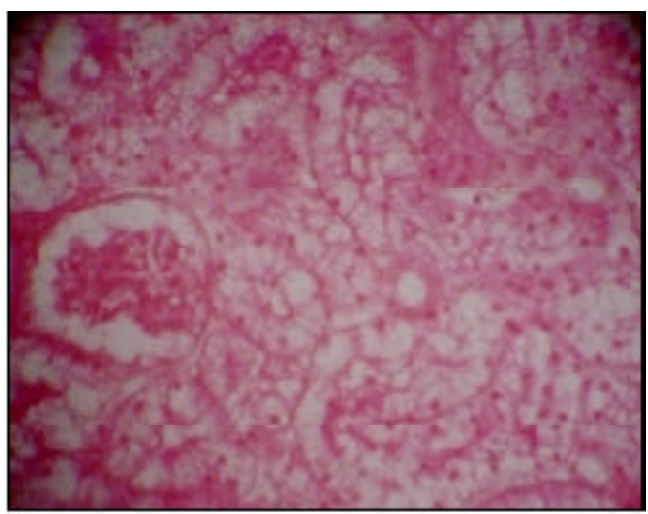

(b)

Figura 3. Riñón de equino con síndrome ulceroso gástrico en grado 4, según la clasificación de Merritt (2003). (a) Congestión severa. Pérdida de la relación córticomedular, necrosis cortical, necrosis tubular aguda, necrosis papilar confluente. (b) Congestión renal severa y hemorragia, degeneración hidrópica severa, presencia de cilindros hialinos hematúricos, nefritis intersticial linfoplasmocítica, glomérulonefritis membranosa, necrosis papilar en unión córtico-medular, necrosis tubular aguda (H\&E, 20X).

\section{Resultados y Discusión}

Los resultados de la evaluación macroscópica y microscópica de la mucosa gástrica indicaron que $6,6,12$, y 6 equinos presentaban lesiones con grados 1, 2, 3 y 4 , respectivamente (Fig. 1). Los cortes histológicos revelaron, además, grados variables de exposición del corion, acompañados de infiltrado mononuclear linfocítico y plasmocítico en la lámina propia; e hiperqueratosis paraqueratósica severa, con fibroplasia focal.

La evaluación macroscópica del hígado evidenció ictericia en 22 animales, hepatomegalia con patrón lobulillar en 18 casos, y focos de necrosis de coagulación confluente con respuesta micronodular y fibroplasia focalizada en todos los casos (Fig. 2a). Los cortes histológicos evidenciaron ictericia en 22 casos, necrosis periacinar en 25 casos, respuesta micronodular en 16 casos, y fibrosis hepática segmental en 12 casos
(Fig. 2b). Asimismo, degeneración grasa en todos los casos.

El examen macroscópico de riñón mostró congestión severa en todos los casos. En el examen microscópico se observó necrosis tubular aguda en todos los casos, pérdida de la relación córticomedular en 20 casos, necrosis cortical en 18 casos, necrosis papilar confluente en 6 casos, y adherencia de la cápsula con fibroplasia intersticial en 4 casos (Fig. 3). Los cortes histológicos mostraron congestión renal severa y hemorragia, degeneración hidrópica y necrosis tubular aguda en todos los animales, necrosis papilar en unión córticomedular en 20 casos, presencia de cilindros hialinos hematúricos en 14 casos, nefritis intersticial linfoplasmocitica en 10 casos, y glomérulonefritis membranosa en 15 casos (Fig. 3b).

La mucosa gástrica, el hígado y los riñones sufren importantes cambios en el caballo de carrera bajo las severas condiciones de entrenamiento. Las lesiones gástricas ob- 
servadas corresponden con el síndrome ulceroso gástrico. Las lesiones hepáticas evidencian una hepatopatía tóxica, mientras que el tejido renal evidenció una nefropatía tóxica con síndrome de pérdida de proteínas.

Existen reportes de lesiones gástricas, hepáticas y renales asociadas a terapéuticas prolongadas con anti-inflamatorios (fenilbutazona), diuréticos (furosemida) y mineralcorticoides (dexametasona) (De Novo, 1993; Donald, 1999; Botana et al., 2002; Cotran et al., 2000). En base a la historia clínica de estos animales, las lesiones observadas en la mucosa gástrica, hígado y riñón estarían asociadas con terapéuticas prolongadas con anti-inflamatorios no esteroideos (AINES).

\section{Agradecimientos}

Los autores agradecen al Consejo de Desarrollo Científico y Humanístico de la Ilustre Universidad Central de Venezuela y a la Dirección de Desarrollo y Fomento Equino del Instituto Nacional de Hipódromos «La Rinconada», Caracas, Venezuela.

\section{Literatura Citada}

1. Aluja A, Constantino C. 2002. Technical of necropsy in domestic animals. $2^{\text {nd }} e d$. México: Manual Moderno. $103 \mathrm{p}$.
2. Andrews FM, Buchanan BR, Elliot SB, Clariday NA, Edwards LH. 2005. Gastric ulcers in horses. J Anim Sci 83: 18-21.

3. Banks W. 1996. Applied veterinary histology. $2^{\text {nd }}$ ed. México: Manual Moderno. 492 p.

4. Botana L, Landoni F, Martín T. 2002. Farmacología y terapéutica veterinaria. Madrid: McGraw-Hill. 690 p.

5. Cotran R, Kumar V, Collins T. 2000. Robbins patología estructural y funcional. $6^{\text {a }}$ ed. Madrid: Mac Graw-Hill Interamericana. $377-853 \mathrm{p}$.

6. De Novo $R$. 1993. Medical management of gastritis, ulcers, and erosions. In: XVII Annual Waltham/OSU Symposium for the Treatment of Small Animal Diseases. USA. p 46-50.

7. Donald M. 1999. Special veterinary pathology. $3^{\text {rd }}$ ed. USA: Mosby. 239 p.

8. Merritt A. 2003. The equine stomach: A personal perspective (1963-2003). In: 49 Annual Convention of the American Association of Equine Practitioners. New Orleans, Louisiana, USA.

9. Moyaert H, Decostere A, Vandamme P, Debruyne L, Mast J, Baele M, Ceelen L, Haesebrouck F. 2007. Helicobacter equorum sp. nov., a urease-negative Helicobacter species isolated from horse faeces. Int J Syst Evol Micr 57: 213-218.

10. Murray MJ. 1994. Gastric ulcers in adult horses. Compendium 16: 792-797. 